\title{
EFEITO DE DOSES DE NPK SOBRE OS TEORES DE NUTRIENTES NAS FOLHAS E NO SOLO, E NA PRODUTIVIDADE DO MARACUJAZEIRO AMARELO ${ }^{1}$
}

\author{
ANA LÚCIA BORGES ${ }^{2}$, RANULFO CORREA CALDAS², ADELISE DE ALMEIDA LIMA², ISRAEL ELY DE ALMEIDA
}

\begin{abstract}
RESUMO - Objetivou-se avaliar a influência das adubações com nitrogênio, fósforo e potássio nos teores de nutrientes das folhas do maracujazeiro amarelo (Passiflora edulis f. flavicarpa Deg.) e em propriedades químicas do solo, tendo em vista maximizar a produtividade e otimizar a prática da adubação. O experimento foi conduzido no período de maio/96 a abril/98, em Latossolo Amarelo do Município de Cruz das Almas (BA). Empregou-se o delineamento experimental de blocos casualizados, em esquema fatorial fracionado $1 / 2$ de $4^{3}$, com dois blocos incompletos, avaliando-se quatro doses de $\mathrm{N}\left(0,100,200 \mathrm{e} 300 \mathrm{~kg} \mathrm{ha}^{-1}\right.$ ano $\left.\mathrm{s}^{-1}\right)$, de $\mathrm{P}_{2} \mathrm{O}_{5}\left(0,80,160 \mathrm{e} 240 \mathrm{~kg} \mathrm{ha}^{-1}\right.$ ano $\left.^{-1}\right)$ e de $\mathrm{K}_{2} \mathrm{O}$ $\left(100,300,500\right.$ e $\left.700 \mathrm{~kg} \mathrm{ha}^{-1} \mathrm{ano}^{-1}\right)$. Amostragens de solo e folhas foram realizadas aos 12 e 24 meses após o plantio, bem como foi avaliada a produtividade no primeiro e no segundo ano. A adubação nitrogenada não influenciou os teores de $\mathrm{N}$ na folha, mas diminuiu os de boro e reduziu o pH do solo no segundo ano de cultivo. A adubação fosfatada aumentou, em média, apenas $12 \%$ os teores de $\mathrm{P}$ nas folhas e 35 vezes no solo. A adubação potássica elevou os teores do nutriente nas folhas do maracujazeiro, e no solo a valores acima do nível ótimo. A produtividade máxima de 22,1 t ha ${ }^{-1}$, em dois anos de cultivo, foi obtida com a aplicação de $244 \mathrm{~kg} \mathrm{de} \mathrm{N,72} \mathrm{kg}$ de $\mathrm{P}_{2} \mathrm{O}_{5}$ e $285 \mathrm{~kg} \mathrm{ha}^{-1}$ de $\mathrm{K}_{2} \mathrm{O}$.
\end{abstract}

Termos para indexação: Passiflora edulis Sims f.flavicarpa Deg., nitrogênio, fósforo, potássio, cálcio, magnésio, enxofre, micronutrientes.

\section{EFFECT OF NPK ON NUTRIENT LEVELS IN LEAVES AND SOIL, AND ON YIELD OF YELLOW PASSION FRUIT}

\begin{abstract}
This work evaluated the effect of nitrogen, phosphorus and potassium fertilization on nutrient levels in the leaves of yellow passion fruit (Passiflora edulis f. flavicarpa Deg.) and on the chemical properties of the soil, in order to maximize yield and optimize fertilizer applications. The experiment was carried out from May/96 to April/98, on a Yellow Latosol, in Cruz das Almas city, State of Bahia, Brazil. It was used a randomized block design, in a fractionated factorial arrangement $1 / 2$ of $4^{3}$, with two incompleted blocks. It was evaluated four doses of $\mathrm{N}\left(0,100,200\right.$ and $300 \mathrm{~kg} \mathrm{ha}^{-1}$ year $\left.{ }^{-1}\right)$, of $\mathrm{P}_{2} \mathrm{O}_{5}\left(0,80,160\right.$ and $240 \mathrm{~kg} \mathrm{ha}^{-1}$ year $\left.^{-1}\right)$ and $\mathrm{K}_{2} \mathrm{O}(100,300$, 500 and $700 \mathrm{~kg} \mathrm{ha}^{-1}$ year $\left.^{-1}\right)$. Soil samples and leaves were collected at 12 and 24 months after planting. Yield was evaluated in the first and second production years. Nitrogen fertilization did not affect the $\mathrm{N}$ level in the leaves but decreased boron and reduced the soil $\mathrm{pH}$ during the second production year. Phosphorus fertilization increased, in average, only $12 \%$ of the P levels in the leaves, and 35 times in soil. Potassium fertilization increased nutrient contents in leaves and in soil over the optimum level. Maximum yield of $22 \mathrm{tha}^{-1}$, during two years, was obtained with application of $244 \mathrm{~kg}$ of N, $72 \mathrm{~kg}$ of $\mathrm{P}_{2} \mathrm{O}_{5}$ and $285 \mathrm{~kg}$ of K $\mathrm{O} \mathrm{ha}^{-1}$.
\end{abstract}

Index terms: Passiflora edulis Sims f. flavicarpa Deg., nitrogen, phosphorus, potassium, calcium, magnesium, sulfur, micronutrients.

\section{INTRODUÇÃO}

O nitrogênio e o potássio são os nutrientes mais absorvidos pelo maracujazeiro. O nitrogênio tem função estrutural na planta, sendo fundamental para o crescimento vegetativo e produção (Kliemann et al., 1986; Baumgartner, 1987), estimulando o desenvolvimento de gemas floríferas e frutíferas. Assim, na sua falta, o crescimento da planta é lento, o porte é reduzido, com presença de ramos finos e em menor número (Marteleto, 1991). O potássio está presente na planta na forma iônica, atuando como ativador enzimático e participando de vários processos (Malavolta et al., 1989). A deficiência desse nutriente reduz o peso da planta e a produção de frutos, além de interferir na qualidade dos frutos e do suco. Apesar de o maracujazeiro absorver pouco fósforo, este é um nutriente importante nos processos de armazenamento e transferência de energia. Na sua ausência, o crescimento da planta é reduzido e a produção de frutos afetada (Baumgartner, 1987).

Quanto à resposta do maracujazeiro aos nutrientes, alguns trabalhos apresentam-se contraditórios. Assim é que Baumgartner et. al. (1978) obtiveram resposta positiva ao nitrogênio, fósforo e potássio no primeiro ano de produção e ao nitrogênio e fósforo no segundo ano, enquanto que nos trabalhos de Müller et. al. (1979), Colauto et al. (1986), Faria et al. (1987) e Borges et al. (1998), o maracujazeiro não respondeu em produtividade à aplicação de NPK no solo.

O equilíbrio nutricional durante o ciclo do maracujazeiro é importante para se obter altas produtividades. Para avaliação do comportamento do maracujazeiro, a associação das análises químicas do solo e a diagnose foliar, vem se mostrando útil na

1 (Trabalho 066/2001). Recebido: 16/03/2001. Aceito para publicação: 27/11/2001. Trabalho realizado com recursos do Convênio Embrapa/ Petrobras.

2 Eng $^{\circ}$ Agr $^{\circ}$, Pesquisador da Embrapa Mandioca e Fruticultura, CEP: 44380-000 Cruz das Almas-BA.

3 Estudante de agronomia da UFBA, bolsista Pibic-CNPq/Embrapa,CEP: 44380-000 Cruz das Almas-BA. 
consecução deste objetivo, por permitir a correlação das doses de nutrientes aplicadas no solo com os teores dos mesmos na planta, como também com sua produtividade.

Também em relação aos teores de nutrientes nas folhas do maracujazeiro, verifica-se grande variação, segundo dados apresentados por diferentes autores (Haag et al., 1973; Malavolta et al., 1989; IFA, 1992). Estas diferenças podem ser ocasionadas por razões como época de amostragem, idade da folha, variedade, condições de desenvolvimento da planta, manejo, teor de nutriente no solo etc. Contudo, para folhas adultas, totalmente desenvolvidas, coletadas em plantas vigorosas, considera-se como adequados os seguintes teores: $\mathrm{N}=47,5-52,5 \mathrm{~g} \mathrm{~kg}^{-1} ; \mathrm{P}=$ $2,5-3,5 \mathrm{~g} \mathrm{~kg}^{-1} ; \mathrm{K}=20-25 \mathrm{~g} \mathrm{~kg}^{-1} ; \mathrm{Ca}=5-15 \mathrm{~g} \mathrm{~kg}^{-1} ; \mathrm{Mg}=2,5-3,5 \mathrm{~g} \mathrm{~kg}^{-}$ ${ }^{1} ; \mathrm{S}=2,0-4,0 \mathrm{~g} \mathrm{~kg}^{-1} ; \mathrm{B}=25-100 \mathrm{mg} \mathrm{kg}^{-1} ; \mathrm{Cu}=5-20 \mathrm{mg} \mathrm{kg}^{-1} ; \mathrm{Fe}=$ $100-200 \mathrm{mg} \mathrm{kg}^{-1} ; \mathrm{Mn}=50-200 \mathrm{mg} \mathrm{kg}^{-1} \mathrm{e} \mathrm{Zn}=45-80 \mathrm{mg} \mathrm{kg}^{-1}$ (IFA, 1992). No Brasil, Haag et al. (1973), em condições de campo, para maracujá amarelo, constataram os seguintes teores de macronutrientes, $\mathrm{em} \mathrm{g} \mathrm{kg}^{-1}$ : $\mathrm{N}=36-46 ; \mathrm{P}=2,1-3,0 ; \mathrm{K}=23,6-32,4$; $\mathrm{Ca}=17,4-27,7 ; \mathrm{Mg}=2,1 \mathrm{e} \mathrm{S}=4,4 ; \mathrm{e}$ os de micronutrientes, em mg $\mathrm{kg}^{-1}: \mathrm{B}=39-47 ; \mathrm{Cu}=15-16 ; \mathrm{Fe}=116-233 ; \mathrm{Mn}=433-604 \mathrm{e} \mathrm{Zn}=26-$ 49 .

Objetivou-se neste trabalho avaliar a influência das adubações com nitrogênio, fósforo e potássio sobre os teores de nutrientes nas folhas do maracujazeiro amarelo (Passiflora edulis Sims. f. flavicarpa Deg.) e as propriedades químicas do solo, tendo em vista maximizar a produtividade e otimizar a prática da adubação.

\section{MATERIAL E MÉTODOS}

O experimento foi conduzido no período de maio de 1996 a abril de 1998, no Município de Cruz das Almas (BA), região do Recôncavo Baiano, cujo clima é subúmido, com temperatura média anual de $24^{\circ} \mathrm{C}$. A pluviosidade de maio/96 a abril/97 foi de $1.483,2 \mathrm{~mm}$ e de maio/97 a abril/98 de $910,7 \mathrm{~mm}$.

O solo da área experimental é um Latossolo Amarelo, franco argilo arenoso ( $620 \mathrm{~g}$ de areia, $90 \mathrm{~g}$ de silte e $290 \mathrm{~g}$ de argila $\mathrm{kg}^{-1}$ ), apresentando, na camada de $0-20 \mathrm{~cm}$ de profundidade, as seguintes propriedades químicas: $\mathrm{pH}$ em água $=4,5 ; \mathrm{P}$ (Mehlich) $=2,0 \mathrm{mg} \mathrm{dm}^{-3} ; \mathrm{K}=78 \mathrm{mg} \mathrm{dm}^{-3} ; \mathrm{Ca}=0,8 \mathrm{cmol}_{\mathrm{c}} \mathrm{dm}^{-3} ; \mathrm{Mg}=0,4 \mathrm{cmol}_{\mathrm{c}}$ $\mathrm{dm}^{-3} ; \mathrm{Al}=0,5 \mathrm{cmol}_{\mathrm{c}} \mathrm{dm}^{-3} ; \mathrm{SB}=1,5 \mathrm{cmol}_{\mathrm{c}} \mathrm{dm}^{-3}, \mathrm{~V}=29 \%$ e matéria orgânica $=17 \mathrm{~g} \mathrm{~kg}^{-1}$.

Utilizou-se o delineamento experimental de blocos casualizados, em esquema fatorial fracionado $1 / 2 \mathrm{de} 4^{3}$, com dois blocos incompletos de 16 tratamentos, avaliando-se quatro doses de $\mathrm{N}\left(0,100,200\right.$ e $300 \mathrm{~kg} \mathrm{ha}^{-1}$ ano $\left.^{-1}\right)$, de $\mathrm{P}_{2} \mathrm{O}_{5}(0,80,160$ e $240 \mathrm{~kg}$ ha $^{-1}$ ano $\left.^{-1}\right)$ e de $\mathrm{K}_{2} \mathrm{O}\left(100,300,500\right.$ e $700 \mathrm{~kg} \mathrm{ha}^{-1}$ ano $\left.^{-1}\right)$. O maracujá amarelo foi plantado em 20/05/96, no espaçamento $2,5 \mathrm{~m} \mathrm{x} \mathrm{5,0} \mathrm{m}$ (800 plantas/ha), com parcela útil de $75 \mathrm{~m}^{2}$, constando de seis plantas, em duas linhas, sendo a parcela total de 20 plantas em $250 \mathrm{~m}^{2}$, com bordadura dupla, ocupando uma área total de 8.000 $\mathrm{m}^{2}$ (640 plantas). O experimento foi irrigado por microaspersão e monitorado por tensiômetros. A polinização foi natural no primeiro ano e manual a partir do $12^{\circ}$ mês.

A calagem foi realizada antes do plantio, em toda a área, utilizando-se 3,4 t de calcário dolomítico, com PRNT de $80 \%$, visando elevar a saturação por bases para $80 \%$. O fósforo, na forma de superfosfato simples, foi parcelado três vezes no ano, sendo um terço na cova de plantio, juntamente com os micronutrientes (50 g de FTE BR12) e os dois terços restantes no quinto e nono mês. Os micronutrientes foram repetidos no ano seguinte, enquanto o nitrogênio, na forma de uréia, e o potássio, como cloreto, foram aplicados em cobertura, a cada dois meses. Aos 17 meses, devido ao aparecimento de superbrotamento em $56 \%$ das plantas, suspeitando-se de deficiência de boro, foram aplicados 12,5 g de bórax/planta, bem como foi realizada a poda dos ramos com problemas.

As amostragens de solo e folha foram realizadas aos 12 e 24 meses após o plantio, coletando-se o solo na profundidade de $0-20 \mathrm{~cm}$ (uma amostra de cada lado das seis plantas úteis), formando-se uma amostra composta por tratamento. Para a análise foliar, coletou-se a quarta folha (duas folhas/planta útil) a partir da extremidade, de ramos medianos sem frutos, obtendose uma amostra composta por tratamento. Após o beneficiamento das amostras, procedeu-se a análise química para determinação dos teores de nutrientes.

A produtividade obtida refere-se às colheitas realizadas três vezes por semana, no período de dezembro/96 a abril/97 (primeiro ano) e maio/97 a abril/98 (segundo ano), cujos dados foram analisados estatisticamente pela análise de variância (teste F) e regressão polinomial. Ajustes de superfícies de respostas foram feitos para os nutrientes, dando-se ênfase aos teores de fósforo e potássio no solo e nitrogênio, fósforo e potássio nas folhas. Foram feitas correlações em doses fixadas de nitrogênio, fósforo e potássio de maior relação benefício/custo marginal (Borges et al., 1999), entre produtividade e os teores de fósforo e potássio no solo e nitrogênio, fósforo e potássio nas folhas do maracujazeiro.

\section{RESULTADOS E DISCUSSÃO}

Os valores de F são apresentados na Tabela 1, mostrando significância dos parâmetros lineares para as variáveis fósforo e potássio no solo e potássio nas folhas, no primeiro e segundo anos de produção. A inexistência de valores significativos para a maioria dos parâmetros quadráticos e de produtos cruzados não possibilitou que as superfícies de respostas estimadas apresentassem pontos de máxima ou de mínima para as variáveis analisadas.

Os teores foliares aos 12 meses mostraram valores de $\mathrm{N}$ superiores aos constatados por Haag et al. (1973) e IFA (1992); e aos 24 meses os teores de $\mathrm{N}$ se encontraram na faixa proposta pelo IFA (1992) mas ainda superiores aos obtidos por Haag et al. (1973) (Tabela 2). Além disso, observou-se aumento máximo dos teores de apenas $2,5 \%$ e $1,8 \%$ com o acréscimo das doses de $\mathrm{N}$ aplicadas no solo.

Os teores de fósforo $(\mathrm{P})$ nas folhas aumentaram no máximo $9 \%$ no primeiro ano e $14 \%$ no segundo ano, com as doses aplicadas desse nutriente (Tabela 2). Os valores obtidos estão adequados, de acordo com IFA (1992) e acima dos obtidos por Haag et al. (1973), principalmente no primeiro ano (Tabela 2). Observou-se correlação significativa apenas no segundo ano entre teores de $\mathrm{P}$ no solo e nas folhas, tanto na dose de $80 \mathrm{~kg} / \mathrm{ha}$ de $\mathrm{P}_{2} \mathrm{O}_{5}$, quanto de $100 \mathrm{~kg} / \mathrm{ha}$ de $\mathrm{K}_{2} \mathrm{O}$ (Tabela 3).

Os teores de potássio $(\mathrm{K})$ encontraram-se acima do recomendado pelo IFA (1992) e aumentaram em média 7,5\% com 
TABELA 1 - Valores de F para os parâmetros estimados da superfície de resposta para as variáveis, fósforo no solo (PS), potássio no solo (KS), nitrogênio nas folhas (NF), fósforo nas folhas (PF) e potássio nas folhas (KF), no primeiro e segundo ano de produção, na cultura do maracujá amarelo. Cruz das Almas-BA, 1997-1998.

\begin{tabular}{|c|c|c|c|c|c|c|c|c|c|c|c|}
\hline Regressão & GL & \multicolumn{2}{|c|}{$\mathrm{PS}\left(\mathrm{mg} \mathrm{dm}^{-3}\right)$} & \multicolumn{2}{|c|}{$\mathrm{KS}\left(\mathrm{mg} \mathrm{dm}^{-5}\right)$} & \multicolumn{2}{|c|}{$\mathrm{NF}\left(\mathrm{g} \mathrm{kg}^{-1}\right)$} & \multicolumn{2}{|c|}{ PF $\left(\mathrm{g} \mathrm{kg}^{-1}\right)$} & \multicolumn{2}{|c|}{$\mathrm{KF}\left(\mathrm{g} \mathrm{kg}^{-1}\right)$} \\
\hline Linear $(\mathrm{N}, \mathrm{P}, \mathrm{K})$ & 3 & $11,47^{* *}$ & $7,99 * *$ & $42,79 * *$ & $39,68 * *$ & $0,54 \mathrm{~ns}$ & $1,44 \mathrm{~ns}$ & $0,44 \mathrm{~ns}$ & $1,38 \mathrm{~ns}$ & $4,22 *$ & $3,50 *$ \\
\hline Quadrática $\left(\mathrm{N}^{2}, \mathrm{~K}^{2}, \mathrm{P}^{2}\right)$ & 3 & $1,56 \mathrm{~ns}$ & $3,94 *$ & $0,38 \mathrm{~ns}$ & $3,15 *$ & $2,36 \mathrm{~ns}$ & $0,28 \mathrm{~ns}$ & $0,42 \mathrm{~ns}$ & $1,44 \mathrm{~ns}$ & $0,37 \mathrm{~ns}$ & $0,37 \mathrm{~ns}$ \\
\hline $\begin{array}{l}\text { Produtos cruzados }(\mathrm{NxP}, \\
\text { NxK, PxK) }\end{array}$ & 3 & $0,14 \mathrm{~ns}$ & $0,33 \mathrm{~ns}$ & $3,40 *$ & $0,19 \mathrm{~ns}$ & $2,35 \mathrm{~ns}$ & $5,45 * *$ & $1,67 \mathrm{~ns}$ & $1,09 \mathrm{~ns}$ & $0,92 \mathrm{~ns}$ & $0,71 \mathrm{~ns}$ \\
\hline Média & & 45,6 & 94,0 & 569,1 & 377,2 & 56,9 & 51,0 & 3,3 & 3,0 & 32,6 & 30,2 \\
\hline
\end{tabular}

$* * \mathrm{p} \leq 0,01 ; * \mathrm{p} \leq 0,05 ; \mathrm{ns}-$ não significativo.

TABELA 2 - Teores de nitrogênio $(\mathrm{N})$, fósforo $(\mathrm{P})$, potássio $(\mathrm{K})$, boro $(\mathrm{B})$ e zinco $(\mathrm{Zn})$ nas folhas e produtividade do maracujá amarelo aos 12 e 24 meses de idade, em função das doses de nitrogênio, fósforo e potássio. Cruz das Almas- BA, 1997-1998.

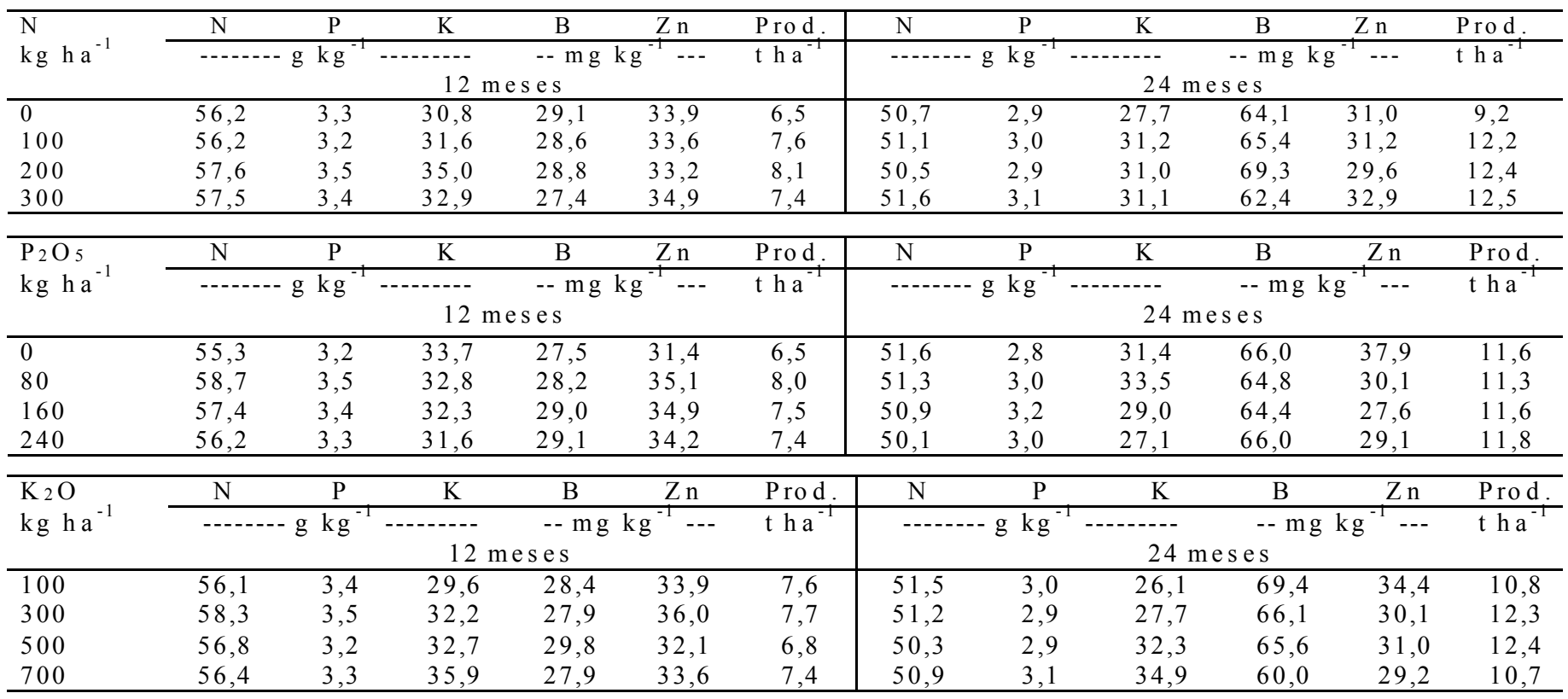

TABELA 3 - Coeficientes de correlação linear simples nas doses de $200 \mathrm{~kg} / \mathrm{ha}$ de N (N200), $80 \mathrm{~kg} / \mathrm{ha} \mathrm{de} \mathrm{P}_{2} \mathrm{O}_{5}(\mathrm{P} 80)$ e $100 \mathrm{~kg} / \mathrm{ha}$ de $\mathrm{K}_{2} \mathrm{O}$ (K100), entre produtividade e teores de fósforo e potássio no solo e nitrogênio, fósforo e potássio nas folhas, aos 12 e 24 meses. Cruz das Almas, BA, 1997-1998.

\begin{tabular}{|c|c|c|c|}
\hline C o rre la çã o & $\mathrm{N} 200$ & P 80 & K 1000 \\
\hline \multicolumn{4}{|c|}{ 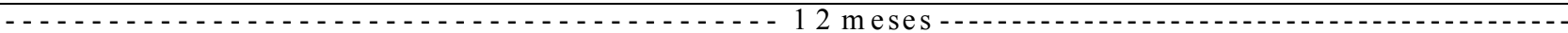 } \\
\hline $\mathrm{P}$ solo $\mathrm{x} N$ fo lha & & $-0,56^{a}$ & $0,73 *$ \\
\hline $\mathrm{P}$ so lo $\mathrm{x} P$ fo lha & & & $0,80 * *$ \\
\hline$K$ solo $x$ P folha & & $0,55^{\mathrm{a}}$ & \\
\hline$K$ solo $x K$ fo lha & & & $0,77 *$ \\
\hline $\mathrm{N}$ fo lha $\mathrm{x} P$ fo lha & $0,69 *$ & $0,66 *$ & $0,64 *$ \\
\hline $\mathrm{N}$ fo lha $\mathrm{x} \mathrm{K}$ fo lha & & $0,58^{a}$ & \\
\hline$P$ fo lha $x K$ folha & & $0,76 *$ & \\
\hline \multicolumn{4}{|c|}{ - - } \\
\hline P rodutividade $\mathrm{x} P$ so lo & & & $0,71 *$ \\
\hline P rodutividade $x$ P fo lha & & & $0,60^{\mathrm{a}}$ \\
\hline Produtivid a d e $x \mathrm{~K}$ fo lha & & & $0,68 *$ \\
\hline $\mathrm{P}$ so lo $\mathrm{x} N$ fo lha & & $0,82 * *$ & \\
\hline $\mathrm{P}$ solo $\mathrm{x} P$ fo lha & & $0,66 *$ & $0,82 * *$ \\
\hline $\mathrm{K}$ so lo $\mathrm{x} P$ fo lha & & & $-0,53^{a}$ \\
\hline $\mathrm{K}$ solo $\mathrm{x} K$ fo lha & $0,51^{\mathrm{a}}$ & & \\
\hline $\mathrm{N}$ fo lha $\mathrm{x} P$ fo lha & $0,78 *$ & $0,84 * *$ & \\
\hline $\mathrm{N}$ fo lha $\mathrm{x} K$ follha & $0,73 *$ & & \\
\hline$P$ fo lha $x K$ fo lha & $0,60^{\mathrm{a}}$ & $0,53^{\mathrm{a}}$ & $0,65 *$ \\
\hline
\end{tabular}

**Significativa a $1 \%$ de probabilidade; *Significativa a $5 \%$ de probabilidade e a Significativa a $\leq 10 \%$ de probabilidade. 
TABELA 4 - Produtividade $\left(\mathrm{t} \mathrm{ha}^{-1}\right)$ do maracujá amarelo em dois anos de cultivo, nos 32 tratamentos de NPK Cruz das Almas-BA, 19971998.

\begin{tabular}{|c|c|c|c|c|c|}
\hline \multicolumn{3}{|c|}{ Tratamento $\left(\mathrm{kg} \mathrm{ha}^{-1} \mathrm{ano}^{-1}\right)$} & \multicolumn{3}{|c|}{ Produtividade $\left(\mathrm{t} \mathrm{ha}^{-1}\right)$} \\
\hline $\mathrm{N}$ & $\mathrm{P}_{2} \mathrm{O} 5$ & $\mathrm{~K}_{2} \mathrm{O}$ & $1997\left(1 \frac{0}{\text { ano }}\right)$ & $1998\left(2^{\circ}\right.$ ano $)$ & $\sum\left(1^{\circ}\right.$ e $2^{\circ}$ ano $)$ \\
\hline 0 & 0 & 100 & 2,6 & 7,2 & 9,8 \\
\hline 0 & 0 & 700 & 7,4 & 9,3 & 16,7 \\
\hline 0 & 80 & 300 & 6,3 & 9,9 & 16,2 \\
\hline 0 & 80 & 500 & 10,2 & 6,4 & 16,6 \\
\hline 0 & 160 & 300 & 8,0 & 12,6 & 20,6 \\
\hline 0 & 160 & 500 & 4,7 & 7,6 & 12,3 \\
\hline 0 & 240 & 100 & 6,1 & 8,9 & 15,0 \\
\hline 0 & 240 & 700 & 6,5 & 11,6 & 18,1 \\
\hline 100 & 0 & 300 & 6,4 & 9,5 & 15,9 \\
\hline 100 & 0 & 500 & 7,3 & 18,6 & 25,9 \\
\hline 100 & 80 & 100 & 3,7 & 7,8 & 11,5 \\
\hline 100 & 80 & 700 & 7,6 & 10,3 & 17,9 \\
\hline 100 & 160 & 100 & 11,5 & 13,6 & 25,1 \\
\hline 100 & 160 & 700 & 7,3 & 10,0 & 17,3 \\
\hline 100 & 240 & 300 & 9,0 & 13,5 & 22,5 \\
\hline 100 & 240 & 500 & 7,6 & 14,0 & 21,6 \\
\hline 200 & 0 & 300 & 7,8 & 12,4 & 20,2 \\
\hline 200 & 0 & 500 & 5,4 & 10,8 & 16,2 \\
\hline 200 & 80 & 100 & 12,6 & 18,0 & 30,6 \\
\hline 200 & 80 & 700 & 5,0 & 9,4 & 14,4 \\
\hline 200 & 160 & 100 & 7,6 & 10,6 & 18,2 \\
\hline 200 & 160 & 700 & 11,5 & 11,1 & 22,6 \\
\hline 200 & 240 & 300 & 7,1 & 12,2 & 19,3 \\
\hline 200 & 240 & 500 & 7,8 & 14,3 & 22,1 \\
\hline 300 & 0 & 100 & 9,1 & 10,9 & 20,0 \\
\hline 300 & 0 & 700 & 6,4 & 13,7 & 20,1 \\
\hline 300 & 80 & 300 & 12,8 & 19,1 & 31,9 \\
\hline 300 & 80 & 500 & 5,9 & 9,5 & 15,4 \\
\hline 300 & 160 & 300 & 4,3 & 9,3 & 13,6 \\
\hline 300 & 160 & 500 & 5,3 & 18,0 & 23,3 \\
\hline 300 & 240 & 100 & 8,0 & 9,5 & 17,5 \\
\hline 300 & 240 & 700 & 7,4 & 10,3 & 17,7 \\
\hline \multicolumn{3}{|c|}{ M é dia } & 7,4 & 11,6 & 18,9 \\
\hline \multicolumn{3}{|c|}{ C V (\%) } & 26,8 & 27,5 & 27,1 \\
\hline \multicolumn{3}{|c|}{ Teste F } & $\mathrm{ns}$ & $\mathrm{ns}$ & $\mathrm{n} \mathrm{s}$ \\
\hline \multirow{2}{*}{\multicolumn{3}{|c|}{$\begin{array}{c}\text { Ponto estimado } \\
N\left(\mathrm{kgha}^{-1}\right)\end{array}$}} & 8,2 (s e la) & 13,6 (s e la) & $22,1(\mathrm{~m}$ á x im o ) \\
\hline & & & 145 & 202 & 244 \\
\hline \multicolumn{3}{|c|}{$\mathrm{P}_{2} \mathrm{O}_{5}\left(\mathrm{~kg} \mathrm{ha}^{-1}\right)$} & 141 & 172 & 72 \\
\hline \multicolumn{3}{|c|}{$\mathrm{K}_{2} \mathrm{O}\left(\mathrm{kgha}^{-1}\right)$} & 514 & 391 & 285 \\
\hline
\end{tabular}

ns - não significativo

as doses aplicadas no solo, tanto aos 12 meses quanto aos 24 meses (Tabela 2). Observou-se correlação positiva entre os teores de $\mathrm{K}$ no solo e nas folhas, notadamente, aos 12 meses, na dose de $100 \mathrm{~kg}$ de $\mathrm{K}_{2} \mathrm{O} / \mathrm{ha}$ (Tabela 3). Vale lembrar que esse é o segundo nutriente mais absorvido pelo maracujazeiro, influenciando no rendimento e na qualidade dos frutos e do suco. Além disso, o K influencia na utilização do $\mathrm{N}$ pelas plantas, de forma que a relação entre os teores foliares de $\mathrm{N}$ e $\mathrm{K}$ tem-se mostrado importante no desenvolvimento das plantas.

O boro (B) encontra-se adequado segundo IFA (1992), mas abaixo dos valores encontrados por Haag et al. (1973), aos 12 meses (Tabela 2). $\mathrm{O}$ aumento de quase três vezes nos teores de $\mathrm{B}$ aos 24 meses (Tabela 2) foi devido à aplicação de bórax aos 17 meses, permanecendo na faixa recomendada por IFA (1992), mas acima dos teores obtidos por Haag et al. (1973). Observouse no primeiro ano uma pequena diminuição do teor de B com o aumento das doses de nitrogênio, provavelmente devido à inibição promovida pelos íons $\mathrm{NO}_{3}^{-} \mathrm{e} \mathrm{NH}_{4}^{+}$sobre a absorção do
$\mathrm{H}_{2} \mathrm{BO}_{3}^{-}$(Malavolta et al., 1989).

Os teores de zinco ( $\mathrm{Zn}$ ) encontram-se abaixo dos recomendados por IFA (1992) e adequados segundo Haag et al. (1973), tanto aos 12 meses quanto aos 24 meses (Tabela 2).

Quanto à produtividade, não se observou significação estatística (Teste F) para as estimativas dos parâmetros das superfícies de respostas relacionadas às doses crescentes de NPK (Tabela 4). Verificaram-se maiores rendimentos nos tratamentos com $300 \mathrm{~kg}$ de N, $80 \mathrm{~kg}$ de $\mathrm{P}_{2} \mathrm{O}_{5}$ e $300 \mathrm{~kg}$ de $\mathrm{K}_{2} \mathrm{O}^{-}$ ${ }^{1}$ e $200 \mathrm{~kg}$ de N, $80 \mathrm{~kg}$ de $\mathrm{P}_{2} \mathrm{O}_{5}$ e $100 \mathrm{~kg} \mathrm{de} \mathrm{K}_{2} \mathrm{O} \mathrm{ha}^{-1}$ (Tabela 4). No entanto, considerando a somatória da produtividade dos dois anos, obteve-se ponto máximo de $22,1 \mathrm{tha}^{-1}$, com a aplicação de $244 \mathrm{~kg}$ de N, $72 \mathrm{~kg}$ de $\mathrm{P}_{2} \mathrm{O}_{5}$ e $285 \mathrm{~kg}$ de $\mathrm{K}_{2} \mathrm{O} \mathrm{ha}^{-1}$. Para esses dois tratamentos as faixas obtidas para os teores foliares, no primeiro ano (12 meses), para os macronutrientes foram, em $\mathrm{g} \mathrm{kg}^{-1}: \mathrm{N}=$ $56,1-58,7 ; \mathrm{P}=3,4-3,5 ; \mathrm{K}=29,6-35,0 ; \mathrm{Ca}=5,5-6,4 ; \mathrm{Mg}=2,9-3,2 \mathrm{e} \mathrm{S}$ $=4,4-4,7$; e para os micronutrientes foram, em $\mathrm{mg} \mathrm{kg}^{-1}: \mathrm{B}=27,9-$ 28,$8 ; \mathrm{Cu}=4,1-4,8 ; \mathrm{Fe}=76,2-83,5 ; \mathrm{Mn}=84,5-96,9 \mathrm{e} \mathrm{Zn}=33,2-36,0$. 
TABELA 5 - Propriedades químicas do solo cultivado com maracujá amarelo aos 12 e 24 meses, na camada de 0 a $20 \mathrm{~cm}$ de profundidade, em função das doses de nitrogênio, fósforo e potássio. Cruz das Almas - BA, 1997-1998.

\begin{tabular}{|c|c|c|c|c|c|c|c|c|}
\hline $\begin{array}{l}\mathrm{N} \\
\mathrm{kg} \mathrm{ha}\end{array}$ & $\begin{array}{c}\mathrm{pH} \\
\mathrm{a} \mathrm{gua}\end{array}$ & $\begin{array}{c}\mathrm{P} \\
---\mathrm{mg} \mathrm{dm}^{-3}{ }^{\mathrm{K}} \\
12 \mathrm{meses} \\
\end{array}$ & $\begin{array}{l}\mathrm{V} \\
\%\end{array}$ & $\begin{array}{l}\mathrm{M} . \mathrm{O}^{2} \\
\mathrm{~g} \mathrm{~kg}\end{array}$ & $\begin{array}{c}\mathrm{pH} \\
\mathrm{a} \mathrm{gua}\end{array}$ & $\begin{array}{r}\mathrm{P} \\
-\mathrm{K} \\
24 \mathrm{meses} \\
\end{array}$ & $\begin{array}{l}\mathrm{V} \\
\%\end{array}$ & $\begin{array}{l}{\mathrm{M} . \mathrm{O}^{2}}^{2} \\
\mathrm{~g} \mathrm{~kg}^{-1}\end{array}$ \\
\hline 0 & 4,6 & $44 \quad 558$ & 49 & 15,2 & 5,1 & $69 \quad 412$ & 56 & 16,8 \\
\hline 200 & 4,6 & 548 & 51 & 16,8 & 4,6 & 107 & 52 & 16,4 \\
\hline 300 & 4,7 & 601 & 51 & 16,7 & 4,5 & 355 & 48 & 16,1 \\
\hline $\begin{array}{l}\mathrm{P}_{2} \mathrm{O}_{5} \\
\mathrm{~kg} \mathrm{ha}^{-1}\end{array}$ & $\begin{array}{c}\mathrm{pH} \\
\text { água }\end{array}$ & $\begin{array}{r}\mathrm{P} \\
-\mathrm{K} \\
12 \mathrm{meses} \\
\end{array}$ & $\begin{array}{l}\mathrm{V} \\
\%\end{array}$ & $\begin{array}{l}\mathrm{M.O.}^{2} \\
\mathrm{~g} \mathrm{~kg}^{-1}\end{array}$ & $\begin{array}{l}\mathrm{pH} \\
\text { água }\end{array}$ & $\begin{array}{r}\mathrm{P} \\
-\mathrm{mg} \mathrm{dm}^{-3} \mathrm{~K} \\
24 \mathrm{meses} \\
\end{array}$ & $\begin{array}{l}\mathrm{V} \\
\%\end{array}$ & $\begin{array}{l}\mathrm{M.O.}^{2} \\
\mathrm{~g} \mathrm{~kg}^{-1}\end{array}$ \\
\hline 0 & 4,6 & $\begin{array}{ll}5 & 662\end{array}$ & 49 & 17,0 & 4,7 & 513 & 48 & 17,4 \\
\hline 80 & 4,6 & 575 & 51 & 17,0 & 5,0 & 349 & 57 & 16,7 \\
\hline 160 & 4,4 & 527 & 49 & 15,4 & 4,6 & 121 & 52 & 16,9 \\
\hline 240 & 4,5 & 107 & 51 & 15,9 & 4,6 & 151 & 51 & 15,9 \\
\hline 300 & 4,5 & 528 & 49 & 15,8 & 4,5 & 362 & 49 & 15,6 \\
\hline 500 & 4,5 & 610 & 49 & 16,1 & 4,8 & 425 & 52 & 16,4 \\
\hline 700 & 4,7 & 873 & 57 & 17,4 & 4,9 & 545 & 58 & 18,4 \\
\hline
\end{tabular}

${ }^{1}$ Soma de bases $(\mathrm{K}+\mathrm{Ca}+\mathrm{Mg}+\mathrm{Na}) ;{ }^{2}$ Matéria orgânica.

No segundo ano ( 24 meses): $\mathrm{N}=50,5-51,5 ; \mathrm{P}=2,9-3,0 ; \mathrm{K}=26,1-$ 33,$5 ; \mathrm{Ca}=10,7,13,8 ; \mathrm{Mg}=4,7-5,6 ; \mathrm{S}=4,8-5,2 ; \mathrm{B}=64,8-69,4 ; \mathrm{Cu}=$ $3,9-4,2 ; \mathrm{Fe}=93,8-100,8 ; \mathrm{Mn}=140,1-199,6 \mathrm{e} \mathrm{Zn}=29,6-34,4$.

Quanto às propriedades químicas do solo, observouse aos 12 meses que não houve decréscimo do $\mathrm{pH}$ com o aumento das doses de $\mathrm{N}$ (Tabela 5). O pH se manteve próximo ao valor inicial $(4,5)$, e vale lembrar que o maracujazeiro é uma planta sensível à acidez (Kliemann et al., 1986). No entanto, foi observado aos 24 meses (Tabela 5) o aumento da acidez, que pode ser atribuído à nitrificação do $\mathrm{NH}_{4}^{+}$proveniente da hidrólise da uréia.

Observou-se naturalmente aumento dos teores de $\mathrm{P}$ no solo com as doses aplicadas (Tabela 5). $\mathrm{O}$ teor médio de $\mathrm{P}$ aumentou em relação ao teor inicial 23 vezes após o primeiro ano e 47 vezes após o segundo ano (Tabela 5), indicando ser um nutriente de pouca mobilidade no solo, apesar de este conter 620 $\mathrm{g}$ de areia $\mathrm{kg}^{-1}$.

Tanto aos 12 meses quanto aos 24 meses (Tabela 5) observaram-se altos teores de potássio (K) no solo, maiores no primeiro ano. Os teores comparativamente mais baixos no segundo ano indicam que, além da absorção pela planta e da exportação pelos frutos, pode ter havido lixiviação do nutriente, apesar de ter chovido $572,5 \mathrm{~mm}$ a mais no primeiro ano de cultivo. Contudo, é importante lembrar que o experimento foi irrigado. Vale ressaltar que, mesmo com a adição de apenas $100 \mathrm{~kg} / \mathrm{ha}$ de $\mathrm{K}_{2} \mathrm{O}$, o teor de $\mathrm{K}$ no solo atingiu valor superior ao recomendado pela Comissão Estadual de Fertilidade do Solo (1989) que é de 90 $\mathrm{mg} \mathrm{dm}{ }^{-3}$.

Os valores de saturação por bases (V\%) aumentaram em média $42 \%$ (29\% para 50\%) aos 12 meses e $44 \%$ (29\% para $52 \%$ ) aos 24 meses (Tabela 5) em relação ao valor inicial (V = $29 \%$ ), mas longe de atingirem os $80 \%$ recomendados pela calagem.

O teor de matéria orgânica parece não ter sido influenciado pelas doses de NPK aplicadas ao solo, verificandose pequeno decréscimo com o cultivo em relação ao teor original.
Em relação ao tratamento correspondente à combinação de $200 \mathrm{~kg}$ de N, $80 \mathrm{~kg}$ de $\mathrm{P}_{2} \mathrm{O}_{5}$ e $100 \mathrm{~kg}$ de $\mathrm{K}_{2} \mathrm{O} \mathrm{ha}^{-1}$, que proporcionou a maior relação benefício/custo marginal (Borges et al., 1999), as propriedades químicas do solo atingiram no primeiro ano (12 meses): $\mathrm{pH}=4,4-4,6 ; \mathrm{P}=26-70 \mathrm{mg} \mathrm{dm}^{-3} ; \mathrm{K}=266$ $575 \mathrm{mg} \mathrm{dm}^{-3} ; \mathrm{Ca}=1,4-1,6 \mathrm{cmol}_{\mathrm{c}} \mathrm{dm}^{-3} ; \mathrm{Mg}=0,5 \mathrm{cmol}_{\mathrm{c}} \mathrm{dm}^{-3} ; \mathrm{Al}=$ $0,3-0,4 \mathrm{cmol}_{\mathrm{c}} \mathrm{dm}^{-3} ; \mathrm{CTC}=6,4-7,2 \mathrm{cmol}_{\mathrm{c}} \mathrm{dm}^{-3} ; \mathrm{V}=43-51 \%$ e matéria orgânica $=16,1-17,0 \mathrm{~g} \mathrm{~kg}^{-1}$. E no segundo ano: $\mathrm{pH}=4,6-5,0 ; \mathrm{P}=$ $87-107 \mathrm{mg} \mathrm{dm}^{-3} ; \mathrm{K}=178-360 \mathrm{mg} \mathrm{dm}^{-3} ; \mathrm{Ca}=2,3-2,5 \mathrm{cmol}_{\mathrm{c}} \mathrm{dm}^{-3} ; \mathrm{Mg}$ $=0,7 \mathrm{cmol}_{\mathrm{c}} \mathrm{dm}^{-3} ; \mathrm{Al}=0,2-0,3 \mathrm{cmol}_{\mathrm{c}} \mathrm{dm}^{-3} ; \mathrm{CTC}=7,3-8,0 \mathrm{cmol}_{\mathrm{c}} \mathrm{dm}^{-}$ 3; $\mathrm{V}=51-57 \%$ e matéria orgânica $=16,4-16,7 \mathrm{~g} \mathrm{~kg}^{-1}$.

O estudo das correlações no primeiro ano não mostrou coeficientes significativos entre produtividade e teores de $\mathrm{P}$ e K no solo e N, P e K nas folhas. Houve correlação positiva entre N e P nas folhas nas doses de $200 \mathrm{~kg}$ de N, $80 \mathrm{~kg}$ de $\mathrm{P}_{2} \mathrm{O}_{5}$ e $100 \mathrm{~kg}$ de $\mathrm{K}_{2} \mathrm{O}$ ha $^{-1}$ (Tabela 3). Correlação linear positiva também foi obtida na dose de $100 \mathrm{~kg}_{\text {de }} \mathrm{K}_{2} \mathrm{O} \mathrm{ha}^{-1}$ entre $\mathrm{K}$ no solo e nas folhas (Tabela 3). Correlação linear negativa foi observada na dose de $80 \mathrm{~kg}$ de $\mathrm{P}_{2} \mathrm{O}_{5}$ ha $^{-1}$ entre $\mathrm{P}$ no solo e $\mathrm{N}$ nas folhas, enquanto na dose de $100 \mathrm{~kg}$ de $\mathrm{K}_{2} \mathrm{O} \mathrm{ha}^{-1}$ a correlação foi positiva, ou seja, aumentando $\mathrm{P}$ no solo aumenta o $\mathrm{N}$ nas folhas, nesta dose de potássio (Tabela 3).

No segundo ano, na dose de $100 \mathrm{~kg}^{\text {de }} \mathrm{K}_{2} \mathrm{O} \mathrm{ha}^{-1}$, quanto maior a produtividade, maiores foram os teores de $\mathrm{P}$ no solo $\mathrm{e}$ nas folhas, bem como o de K nas folhas (Tabela 4). A correlação entre $\mathrm{K}$ no solo e nas folhas na dose de $200 \mathrm{~kg}^{\mathrm{de}} \mathrm{N} \mathrm{ha}^{-1}$ foi mais baixa no segundo ano do que no primeiro. A correlação mais alta foi obtida na dose de $80 \mathrm{~kg}$ de $\mathrm{P}_{2} \mathrm{O}_{5}$ ha $^{-1}$ entre $\mathrm{N}$ e $\mathrm{P}$ nas folhas; enquanto, correlação negativa entre $\mathrm{K}$ no solo e $\mathrm{P}$ nas folhas, na dose de $100 \mathrm{~kg}$ de $\mathrm{K}_{2} \mathrm{O} \mathrm{ha}^{-1}$.

\section{CONCLUSÕES}

1. A adubação nitrogenada não influenciou os teores de 
nitrogênio na folha, diminuiu o de boro e reduziu o $\mathrm{pH}$ do solo no segundo ano de cultivo.

2. A adubação fosfatada aumentou, em média, apenas $12 \%$ os teores de fósforo nas folhas e 35 vezes no solo.

3. A adubação potássica elevou os teores de nutrientes nas folhas do maracujazeiro e no solo a valores acima do nível ótimo. 4. A produtividade máxima de $22,1 \mathrm{tha}^{-1}$, em dois anos de cultivo, foi obtida com a aplicação de $244 \mathrm{~kg}$ de N, $72 \mathrm{~kg} \mathrm{de} \mathrm{P}_{2} \mathrm{O}_{5}$ e $285 \mathrm{~kg}$ de $\mathrm{K}_{2} \mathrm{O} \mathrm{ha}^{-1}$.

\section{REFERÊNCIAS BIBLIOGRÁFICAS}

BAUMGARTNER, J.G. Nutrição e adubação. In: RUGGIERO, C. ed. Maracujá. Ribeirão Preto, UNESP, SP: 1987. p.86-96.

BAUMGARTNER, J.G.; LOURENÇO, R.S.; MALAVOLTA, E. Estudos sobre a nutrição mineral e adubação do maracujazeiro (Passiflora edulis Sims f. flavicarpa Deg.) V. Adubação mineral. Científica, São Paulo, SP. n.6, p.361-367, 1978.

BORGES, A.L.; LIMA, A. de A.; CALDAS, R.C. Nitrogênio, fósforo e potássio na produção e qualidade dos frutos de maracujá amarelo - primeiro ano. Cruz das Almas, BA: Embrapa Mandioca e Fruticultura, 1998. 4p. (EMBRAPA-CNPMF. Pesquisa em Andamento, 66).

BORGES, A.L.; LIMA, A. de A.; CALDAS, R.C. Adubação nitrogenada, fosfatada e potássica para o maracujazeiro amarelo em solo de tabuleiros costeiros. Cruz das Almas, BA: Embrapa Mandioca e Fruticultura, 1999. 4p. (EMBRAPA-CNPMF. Comunicado Técnico, 57).

COLAUTO, N.M.; MANICA, I.; RIBOLDI, J.; MIELNICZUK, J. Efeito do nitrogênio, fósforo e potássio, sobre a produção, qualidade e estado nutricional do maracujazeiro amarelo. Pesquisa Agropecuária Brasileira, Brasília, DF. v.21, n.7, p.691695, 1986.
COMISSÃO ESTADUAL DE FERTILIDADE DO SOLO (Salvador, BA). Manual de adubação e calagem para o Estado da Bahia. Salvador, BA: CEPLAC/EMBRAPA/ EPABA/ NITROFÉRTIL. 1989.176p.

FARIA, J.L.C.; MANICA, I.; COLAUTO, N.M.; STRONSKI, M. do S.; BOEIRA, H. Resposta do maracujazeiro amarelo (Passiflora edulis Sims f. flavicarpa Deg.) à adubação com N, P e K, no segundo, terceiro e quarto anos de produção. Revista Brasileira de Fruticultura, Cruz das Almas, BA. v.9, n.3, p.45-50, 1987.

HAAG, H.P.; OLIVEIRA, G.D.; BORDUCCHI, A.S.; SARRUGE, J.R. Absorção de nutrientes por duas variedades de maracujá. Anais da ESALQ, Piracicaba, n.30, p.267-279, 1973.

IFA (Paris, França). World fertilizer use manual. Limburgerhaf: BASF Agricultural Research Station, 1992. 632p.

KLIEMANN, H.J.; CAMPELO JUNIOR, J.H.; AZEVEDO, J.A. de; GUILHERME, M.R.; GEN, P.J. de C. Nutrição mineral e adubação do maracujazeiro (Passiflora edulis Sims). In: HAAG, H.P., ed. Nutrição mineral e adubação de frutíferas tropicais no Brasil. Campinas, SP: Fundação Cargill, 1986. p.245-284.

MALAVOLTA, E.; VITTI, G.C.; OLIVEIRA, S.A. de. Avaliação do estado nutricional das plantas: princípios e aplicações. Piracicaba, SP: POTAFOS, 1989. 201p.

MARTELETO, L.O. Nutrição e adubação. In: SÃO JOSÉ, A.R.; FERREIRA, F.R.; VAZ, R.L., ed. A cultura do maracujá no Brasil. Jaboticabal, SP: FUNEP, 1991.p.125-237.

MÜLLER, C.H.; PINHEIRO, R.V.R.; CASALI, V.W.D.; OLIVEIRA, L.M. de; MANICA, I.; SOUZA, C.G. de. Efeito de doses de sulfato de amônio e de cloreto de potássio sobre a produtividade e sobre a qualidade de maracujá colhidos em épocas diferentes. Revista Ceres, Viçosa, MG. v.26, n.143, p.48-64. 1979. 